\title{
CHRISTOPH INGENHOVEN
}

\section{Rede anlässlich der Eröffnungsfeier des Oeconomicum der Heinrich-Heine-Universität Düsseldorf am 30. November 2010}

Ich begrüße alle, die Patrick Schwarz-Schütte schon begrüßt hat, und mache es mir insofern etwas einfacher. Lassen Sie mich wenige Sätze zu den vielleicht philosophischen, emotionalen Grundlagen dieses Hauses sagen. Denn heute über die technischen und eher materiellen Dinge des Hauses zu sprechen, wäre sicher falsch und würde zu weit führen.

Wer die Bauordnung dieses Landes kennt, der weiß: es gibt Räume, die zum dauernden Aufenthalt von Menschen nicht bestimmt sind. Ich bin allerdings der Meinung, dass der Mensch als solcher nicht dafür bestimmt ist, sich dauerhaft in geschlossenen Räumen aufzuhalten, und vielleicht ist das eine grundsätzliche Erklärung für die Art von Architektur, die wir machen. Und ich meine, wenn man Häuser baut, wenn man sie bauen muss, wenn man zur Erhaltung der Körperwärme - was die Minimalfunktion wäre tatsächlich des Hauses bedarf, dass man dann sozusagen das notwendige, das sinnvolle Minimum tun sollte und das in einer Atmosphäre, die so angenehm und so leicht und so natürlich wie irgend möglich ist: in Kontakt zur gegebenen Lebenswirklichkeit, also zum Beispiel zum Platz dort draußen, zu der Landschaft, zum See, zu EX LIBRIS, zur Terrasse, zu alledem, was das Haus umgibt. Man kann sich in diesem Haus dem Leben darum herum nicht entziehen. Das ist absichtlich so, und ich glaube, das ist auch gut so, wenn man anspruchsvoll ist.

Wir haben uns als Architekten gefragt, ob unsere Architektur, ob das, was wir hier tun, in irgendeiner direkten Beziehung zu dem Thema dieses Hauses stehen kann. Und ich glaube, dass das, was das Haus leisten soll, was in dem Haus geleistet werden soll, in Hinblick auf die Frage Wettbewerb und Fairness, etwas mit Transparenz, mit Nachvollziehbarkeit, mit Verständlichkeit zu tun hat.

Wir bemühten uns sehr darum, und ich hoffe, dass uns das hier auch gelungen ist, dass diese Architektur verständlich ist, dass sie sich unmittelbar erschließt, dass man keiner großen Erklärung, keiner intensiven Anleitung bedarf, um es in Gebrauch nehmen zu können.

Natürlich gibt es so verschiedene Architekturen, wie es Architekten und Menschen auf dieser Welt gibt, und ohne Menschen, die anderer Meinungen sind, zu nahe zu treten, kann ich sagen, dass unsere Überzeugung die ist, dass Architektur keine darstellende Kunst ist, das heißt, sie stellt nicht das Leben dar, sondern sie ermöglicht Leben in diesen Häusern und dafür muss sie unauffällig oder zumindest zurückgenommen sein. Ich habe in meinem Berufsleben die Erfahrung gemacht, dass Dinge, die weit über das wirklich Notwendige hinausgehen, meist nicht tatsächlich mehr bieten als die Dinge, die sehr 
nah am notwendigen, sinnvollen Minimum, nicht an einem vielleicht künstlerisch begründeten Minimalismus, aber an einem sinnvollen Minimum entlang entwickelt worden sind.

Es geht also um räumliche und materielle und um formale Angemessenheit, und es geht auch darum, dass man ehrlich mit den Menschen, die diese Häuser benutzen und die oft sehr lange in ihnen zubringen, umgeht - what you see is what you get, das heißt, räumlich, materiell und formal soll man die Menschen nicht hinters Licht führen. Es soll tatsächlich alles da liegen, offen und verständlich und einfach. Insofern kann man das Wort Transparenz auch als Architekt als einen politischen Auftrag betrachten. Es ist für mich sowieso das Wort des Jahrzehnts. In diesem Haus sollen sich Menschen auf eine informelle Art und Weise treffen können: informell ist vielleicht auch eine Grundlage unserer Architektur.

Warum? Weil man sich sozusagen, ohne sich zu verabreden, trifft, weil man insgesamt wenig hierarchisch operieren kann und soll in diesem Haus, weil die Fähigkeit entscheidet, nicht die Stellung, die man einnimmt, und nicht die Position. Es ist ein Haus, das den Status des Einzelnen nicht noch durch die entsprechenden Herrschaftssymbole unterstützt.

Das Haus bildet einen Raum. Es tut natürlich das, was jedes Haus tun sollte, es hat einen Mehrraum, wenn man so will, der über das Raumprogramm hinausgegangen ist. Es ist der Raum, in dem wir uns heute aufhalten, der in dem Programm nicht gestanden hat. Es hieß natürlich, dass Erschließungsflächen vorgesehen sind, aber es hieß darin nicht, dass es eine über drei Etagen gehende große Halle geben soll, in der man zusammen kommen kann und die auch den Anspruch enthält, Veranstaltungen machen zu können. Es stand auch nicht im Raumprogramm, dass es eine Terrasse geben soll, eine Terrasse, die den See, der vor der Haustür liegt, das kleine "Seechen“ vor der Haustür, nutzt. Wir haben diesen See geradezu okkupiert. Er war vorher quasi unsichtbar. Er war hinter großen Büschen verborgen. Wir haben das Haus auf eine ehemalige Zufahrtsstraße zur Tiefgarage gesetzt. Wir haben also eigentlich gar kein vorhandenes Grundstück genutzt. Es ist zwar formal und juristisch ein Grundstück, aber es ist architektonisch keines gewesen.

Wir haben versucht, das Haus zum Platz zu orientieren, zu einem Platz, der gar keiner war. Es gibt einen Ort dort, vor der großen Universität- und Landesbibliothek, der wegen des Heinrich-Heine-Denkmals womöglich als der zentrale Ort oder Platz der Universität gilt, der aber natürlich räumlich diesem Anspruch nicht gerecht werden konnte. Das zeigt, wie die Universität, was man bedauerlicherweise sagen muss, in der relativen Geschwindigkeit ihrer Entstehung in den 1970er Jahren, die städtebaulichen Aspekte, die auch emotionalen Aspekte, die im Städtebau liegen, vernachlässigt hat.

Es ist eine Universität, die von außen nicht sichtbar ist, die sich in der Stadt und selbst gegenüber ihren nächsten Nachbarn kaum sichtbar darstellt. Wir haben das, was man daran tun kann, mit diesem relativ begrenzt großen Projekt im Verhältnis zu der Gesamtgröße der Universität, zu tun versucht. Wir haben versucht, eine Brücke vom Platz bis zur Straße zu schlagen. Wir haben uns an der Straße gezeigt, und wir haben am Platz dafür gesorgt, dass mit der Bibliothek zusammen tatsächlich so etwas Ähnliches wie ein Platz entstehen konnte. Es ist selbst hinter dem Haus ein intimer 


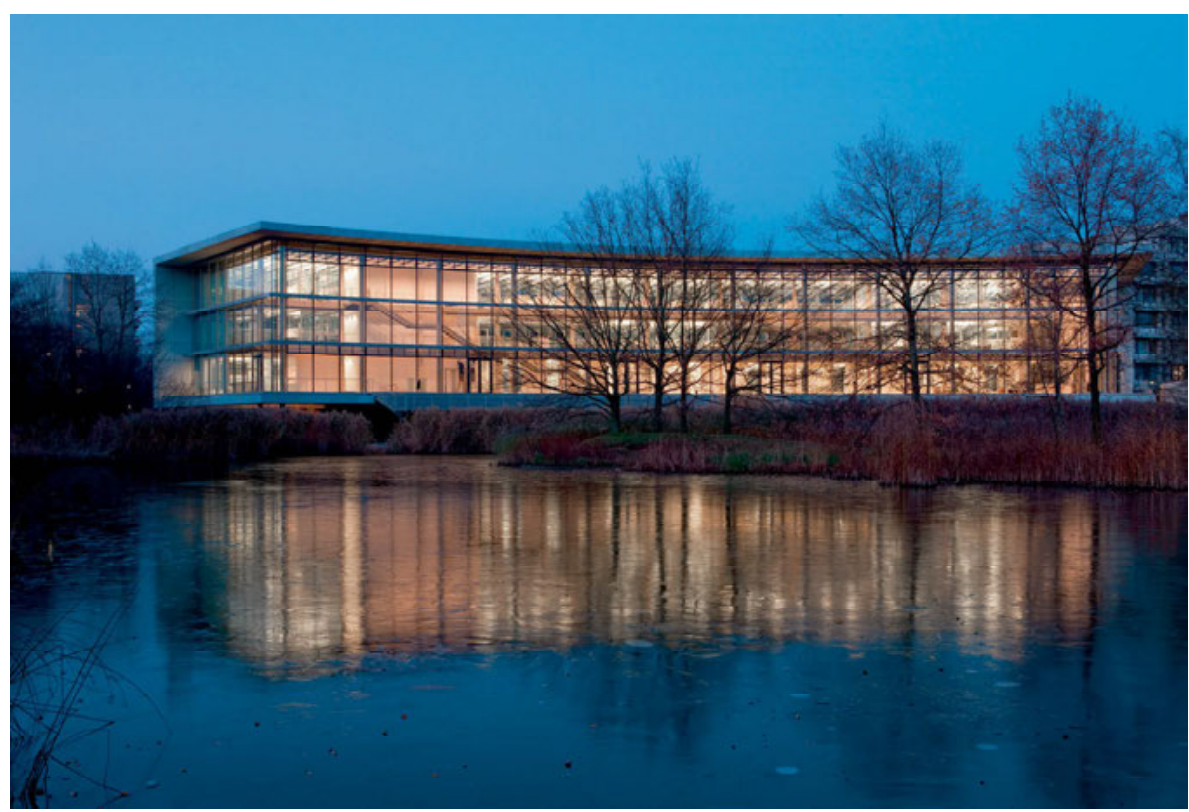

Abb. 1: Blick auf das Oeconomicum vom kleinen See aus

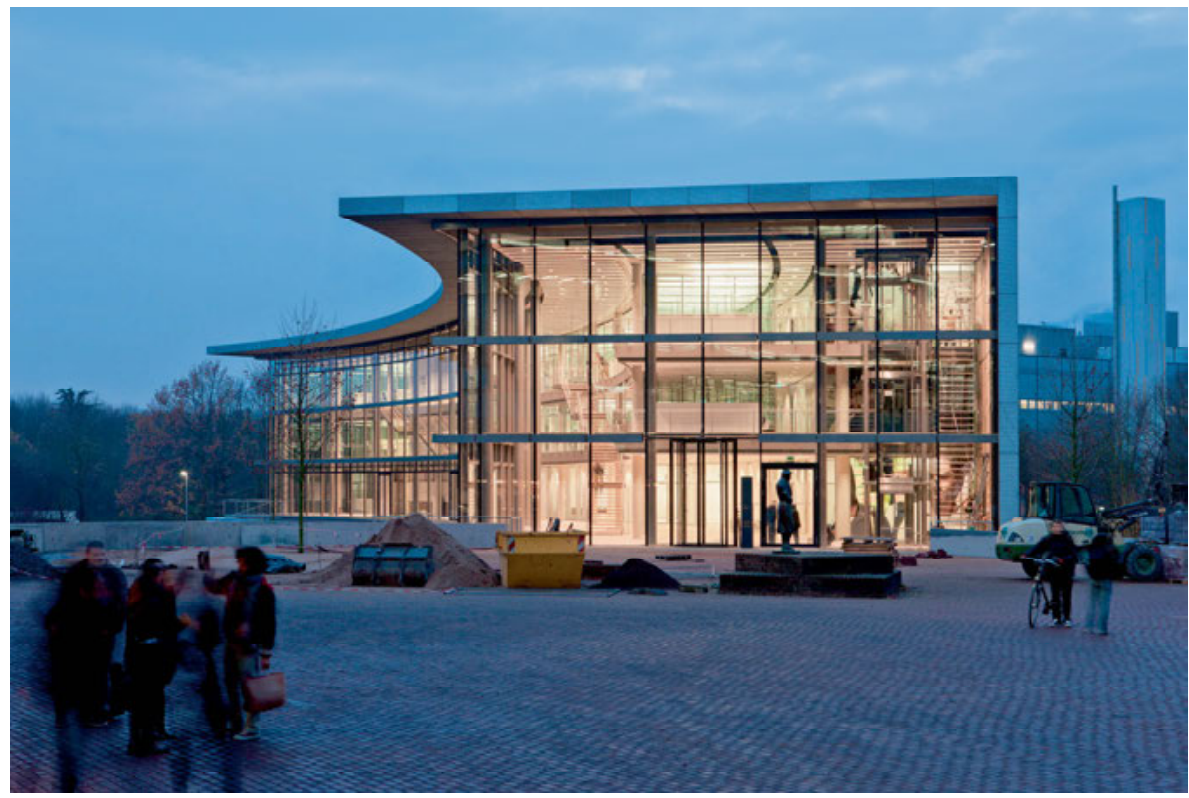

Abb. 2: Blick auf den Eingangsbereich des Oeconomicums 


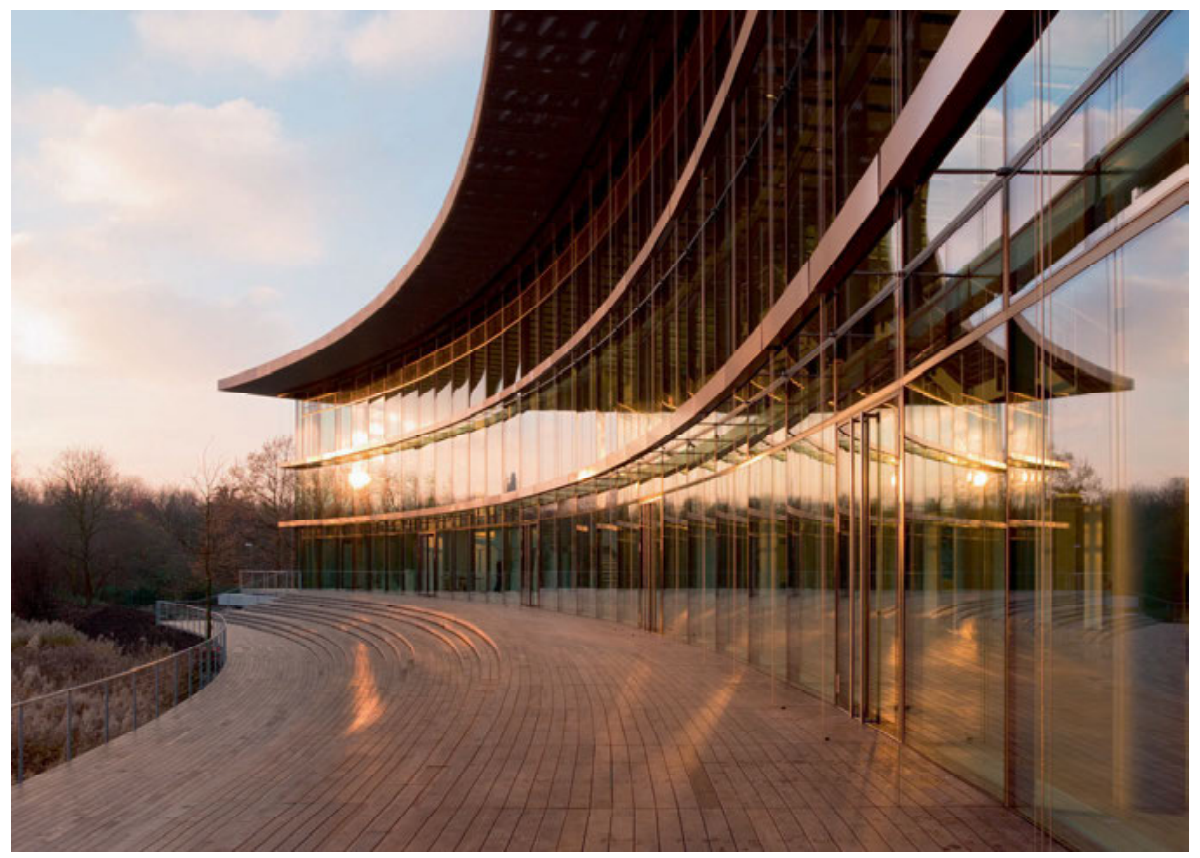

Abb. 3: Terrasse zum See

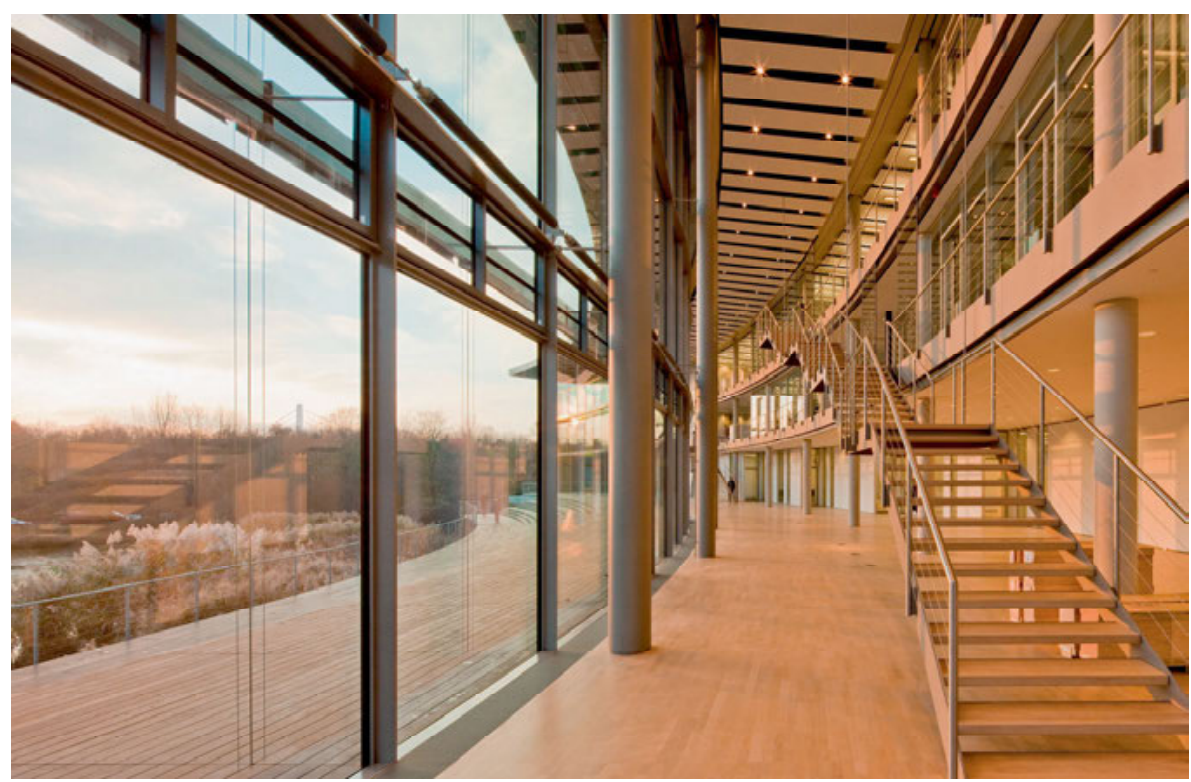

Abb. 4: Übergang von der lichtdurchfluteten Halle zu den Aufgängen 
Garten entstanden; was ursprünglich eine Art Restfläche war, ist heute, wenn man es sich anschaut, des Durchquerens durchaus wert.

Am wichtigsten war uns am Ende das, was man vielleicht - auf Deutsch kann man das kaum ausdrücken - community space nennt, also die Gemeinschaftsflächen. Das klingt immer ein bisschen nach Wohnheim. Aber worauf wir hinaus wollen, ist der Raum, wo Öffentlichkeit tatsächlich stattfinden kann, im begrenzten Rahmen der Universität und deren Öffentlichkeit oder eben in einem größeren Rahmen.

Es ist ein offen zugängliches Gelände, und mit großer Freude haben wir auf der Baustelle beobachtet, wie viel Bewegung hier überall ist, wie viele junge Menschen das Gelände bevölkern und wo sie in Zukunft hoffentlich auch glücklich und zufrieden studieren können, in diesen Räumen. Und studieren heißt ja im Allgemeinen, nicht nur Wissen mehren, was sicherlich in den Räumlichkeiten geschieht, sondern das heißt auch, sich sozial zu beweisen und in irgendeiner Form zu üben, was man dort zu üben hat, und um das zu ermöglichen, sind, so glaube ich, genügend Flächen geschaffen.

Ich möchte mich zum Schluss vor allen Dingen bei meinem Bauherrn bedanken, bei der Familie Schwarz-Schütte und insbesondere bei Patrick: die Freundschaft hat es also ausgehalten, und ich kann sagen, wenn zwei Choleriker zusammen bauen, gehen sie relativ friedlich miteinander um. Das hat vielleicht etwas damit zu tun, dass man einander in dieser Eigenschaft erkennt und mit ein bisschen Vorsicht behandelt.

Ich möchte Sie bitten, alle die, die dieses Haus bevölkern, 100 Tage Geduld zu haben. Das braucht man mindestens bei einem Gebäude. Das hat man mit vielen neuen Dingen, warum nicht auch mit Gebäuden. Sie haben ihre Fehler, sie haben ihre Macken, sie haben ihre Anfangsschwierigkeiten, und sie haben vor allem ihre Ansprüche.

Also lassen Sie es 100 Tage auf sich wirken und wenn sie dann noch ein Problem haben, dann sprechen wir noch einmal darüber. Und wir alle warten auf den nächsten Sommer. Jeder, der hier die Baustelle im letzten Monat gesehen hat, weiß, dass im Sommer die Verflechtung mit dem, was drum herum ist, noch gelungener sein kann und dass natürlich auch die Wunden, die dieses Gebäude trotz aller Bemühungen in die nähere Umgebung gerissen hat, dass diese Wunden natürlich von der Natur geheilt werden. Und so freue ich mich bereits auf das nächste Frühjahr, wenn auf der Terrasse vielleicht irgendeine gelungene Festivität stattfinden wird. Vielen Dank. 


\section{Prof. Dr. Raimund Schirmeister, Dipl.-Math.}

Raimund Schirmeister ging bis 1992 Lehrtätigkeiten an der Albert-Ludwigs-Universität Freiburg im Breisgau, der Universität Augsburg, der Universität-Gesamthochschule Essen sowie an der Universität zu Köln nach. Seit 1992 ist er Univ.-Professor für Betriebswirtschaftslehre, insbesondere Finanzierung und Investition, an der Heinrich-Heine-Universität Düsseldorf. Er war Prodekan (1999/2000, 2002/2003) und Dekan (2000/2002) der Wirtschaftswissenschaftlichen Fakultät und ist seit 2002 Vorsitzender des Wissenschaftlichen Beirats der Düsseldorf Business School GmbH an der HeinrichHeine-Universität Düsseldorf sowie seit 2009 des MBA Gesundheitsmanagement. Raimund Schirmeister war Prorektor für Planung und Finanzen (2003/2007) sowie Chief Information Officer (CIO) der Heinrich-Heine-Universität Düsseldorf (2005/2007).

Seine Forschungsschwerpunkte sind die Investitionstheorie, das Finanzmanagement, das Finanzmarktorientierte Rechnungswesen, Finanzcontrolling sowie Corporate Governance. 